\title{
Comorbidity of Keratoconus and Fuchs' Corneal Endothelial Dystrophy: A Review of the Literature
}

\author{
Ioanna Mylona loannis Tsinopoulos Nikolaos Ziakas \\ 2nd Department of Ophthalmology, Aristotle University of Thessaloniki, Papageorgiou General Hospital, \\ Thessaloniki, Greece
}

\section{Keywords}

Cornea · Fuchs' dystrophy · Keratoconus

\begin{abstract}
Introduction: Comorbidity of keratoconus (KC) and Fuchs' corneal endothelial dystrophy (FD) has been sporadically reported in the literature since the early nineties. This review summarizes findings from the related literature while examining the possibilities related to the concurrent development of these disorders and the clinical significance for the clinician. Methods: NLM/PubMed, Ovid, Google Scholar, Cornea, and Cochrane database were reviewed for research papers presenting cases of comorbid KC and FD published between January 1990 and July 2019. Results: Fifteen papers which presented 69 cases were included in the study. Most cases were women (56.5\%), involvement was bilateral (59.4\%), and the age range was wide (15-82 years) with a normal distribution. Incidence is expected to be approximately 1 per 100,000. FD is the most frequent comorbid corneal dystrophy diagnosed in $\mathrm{KC}$ patients. There are 4 distinct possibilities for the cooccurrence of KC and FD, the most likely being that of chance. Conclusion: The overall incidence of these disorders found in combination is very low and, in absence of other conclusively proven hypotheses, most likely attributable to chance. The treatment plan should be tailored to the needs of the individual patient, since the diag-
\end{abstract}

nosis can be made in any stage of life and could be part of a more complex presentation that includes another pathology of the cornea or senile cataract. The inclusion of preoperative topography and specular microscopy as routine in cataract preoperative assessment would protect against any postoperative complications in these patients.

(c) 2019 S. Karger AG, Basel

\section{Introduction}

Keratoconus (KC) is a progressive, corneal stromal thinning disorder that leads to corneal ectasia, with irregular myopic astigmatism, central anterior scarring, and reduced vision, appearing early in life and seldom after the age of 35 years. Initially, $\mathrm{KC}$ was believed to be a noninflammatory disorder, yet a number of more recent findings that revealed a significant role of proteolytic enzymes, cytokines, and free radicals in the disease have cast doubts on this classification [1]. The estimated prevalence of $\mathrm{KC}$ in the general population has been reported as ranging from 0.9 to $3.3 \%$ [2].

The etiology of $\mathrm{KC}$ is multifactorial. Genetic factors are suspected to have a significant role in the etiopathogenesis of $\mathrm{KC}$, since a positive family history is frequent in $\mathrm{KC}$. Twin and consanguinity studies also revealed a high percentage of cases among relatives $[3,4]$. The significant het- karger@karger.com

www.karger.com/ore

(C) 2019 S. Karger AG, Base

Karger'?
Ioanna Mylona, MD, MSc

2nd Department of Ophthalmology

Papageorgiou General Hospital, Agiou Pavlou 76

Pavlos Melas, GR-564 29 Thessaloniki (Greece)

milona_ioanna@windowslive.com 
erogeneity of findings has not enabled researchers to reach definitive conclusions; however, indirect evidence for genetic factors come from population studies. According to a recent review, $\mathrm{KC}$ may be more prevalent, have earlier onset, and have greater disease progression in certain Asian and non-Asian ethnicities, particularly Indians, Pakistanis, Middle Easterners, and Polynesians, compared with white populations [5]. There is no conclusive finding as to the relative incidence among males and females in $\mathrm{KC}$, since there is a number of sociodemographic factors that obscure the issue. A recent study noted that women presented for treatment due to $\mathrm{KC}$ later in life and were more likely to have a family history of $\mathrm{KC}$, but disease severity appeared to be the same in both sexes [6]. Environmental factors that have been associated with the disease include atopy, exposure to UV radiation, and eye rubbing, yet their relative importance is unknown [7]. These environmental factors cause oxidative damage to $\mathrm{KC}$ corneas because of the inability of $\mathrm{KC}$ corneas to process reactive oxygen species, which leads to corneal thinning and loss of vision [8]. UV radiation directly promotes the formation of free radicals, while eye rubbing is a mechanical stressor; atopy appears to be associated with more frequent eye rubbing and, thus, can be considered as an indirect pathogenetic mechanism [9].

$\mathrm{KC}$ is a bilateral eye disease, and although patients may present with symptoms only unilaterally, modern diagnostic procedures (corneal topography) will detect complimentary eye pathology in the vast majority of cases; in a related longitudinal study, $50 \%$ of the unaffected eyes in the same patient developed $\mathrm{KC}$ within 16 years of initial presentation [10].

Treatment options for early stages of KC include spectacle correction, soft contact lenses, and rigid gas permeable lenses, while posterior keratoplasty was offered for advanced cases. Deep anterior lamellar keratoplasty was introduced as an alternative with similar visual outcomes and fewer complications [11].

Fuchs' corneal endothelial dystrophy (FD) is the most common among a variety of genetically determined corneal dystrophies, a heterogenous group of noninflammatory diseases leading to corneal endothelial dysfunction [12]. FD is dominantly inherited and clinically characterized by guttate excrescences, representing foci of thickened posterior collagenous layer deposited by dysfunctional corneal endothelial cells between Descemet's membrane and the endothelial monolayer [13]. FD is most commonly associated with alterations in the transcription factor 4 (TFC4) gene, localized on chromosome 18 , which lead to RNA aggrega- tion and toxicity [14]. FD is expressed much more frequently in females for as yet unknown reasons [12]. Typically, FD usually progresses over the length of 10-20 years from an edema of the corneal stroma, epithelium, and subepithelial tissue (bullous keratopathy) to a subepithelial fibrosis. The appearance of nonsenile cataracts is common in FD, leading to faster decompensation of the cornea. Treatment options initially were limited to posterior keratoplasty, but more recently, newer procedures for repairing the posterior surface of the cornea are becoming the treatment of choice. These include deep lamellar endothelial keratoplasty, Descemet stripping endothelial keratoplasty, and Descemet stripping automated endothelial keratoplasty [13].

The cooccurrence of $\mathrm{KC}$ and FD has been sporadically reported since the 1990s $[15,16]$. The purpose of this review is to present all these rare occurrences in the literature and assess whether there are any common pathways to disease, as well as present treatment outcomes and the available options for the clinician.

\section{Methods}

NLM/PubMed, Ovid, Google Scholar, Cornea, and Cochrane database were reviewed for research papers presenting cases of comorbid KC and FD published between January 1990 and July 2019. Papers had to have at least an abstract in English and could be any type of study. Studies to be included in the review were assessed independently by 2 reviewers, and all conclusions were reached by consensus amongst the authors. Search terms used in PubMed were: keratoconus[Title/Abstract] AND (fuch[Title/ Abstract] OR dystrophy[Title/Abstract] OR guttae[Title/ Abstract] OR guttata[Title/Abstract]) AND hasabstract[text] AND English[Lang] AND(“1990/01/01”[PDAT]:"2019/07/01”[PDAT]). This yielded 341 results. All results were screened for eligibility along with results from a manual search strategy that was applied to identify any relevant studies missed by the term searches. The manual search strategy involved looking up relevant references from already identified searches and a Google/Yahoo search with the same terms as the PubMed search. No meta-analysis was performed since the vast majority of studies were case reports or case series, with 2 studies being retrograde assessments of larger sets of patient data. There were 15 eligible studies included in the review (Table 1).

\section{Results}

The literature offers 69 cases of combined KC with FD. Published studies are either case reports, specifically addressing the comorbidity and treatment options, or larger-scale studies, where this comorbidity is reported but not the principal focus of the researchers. In the first category, there are single case reports $[15,17-22]$ and case 


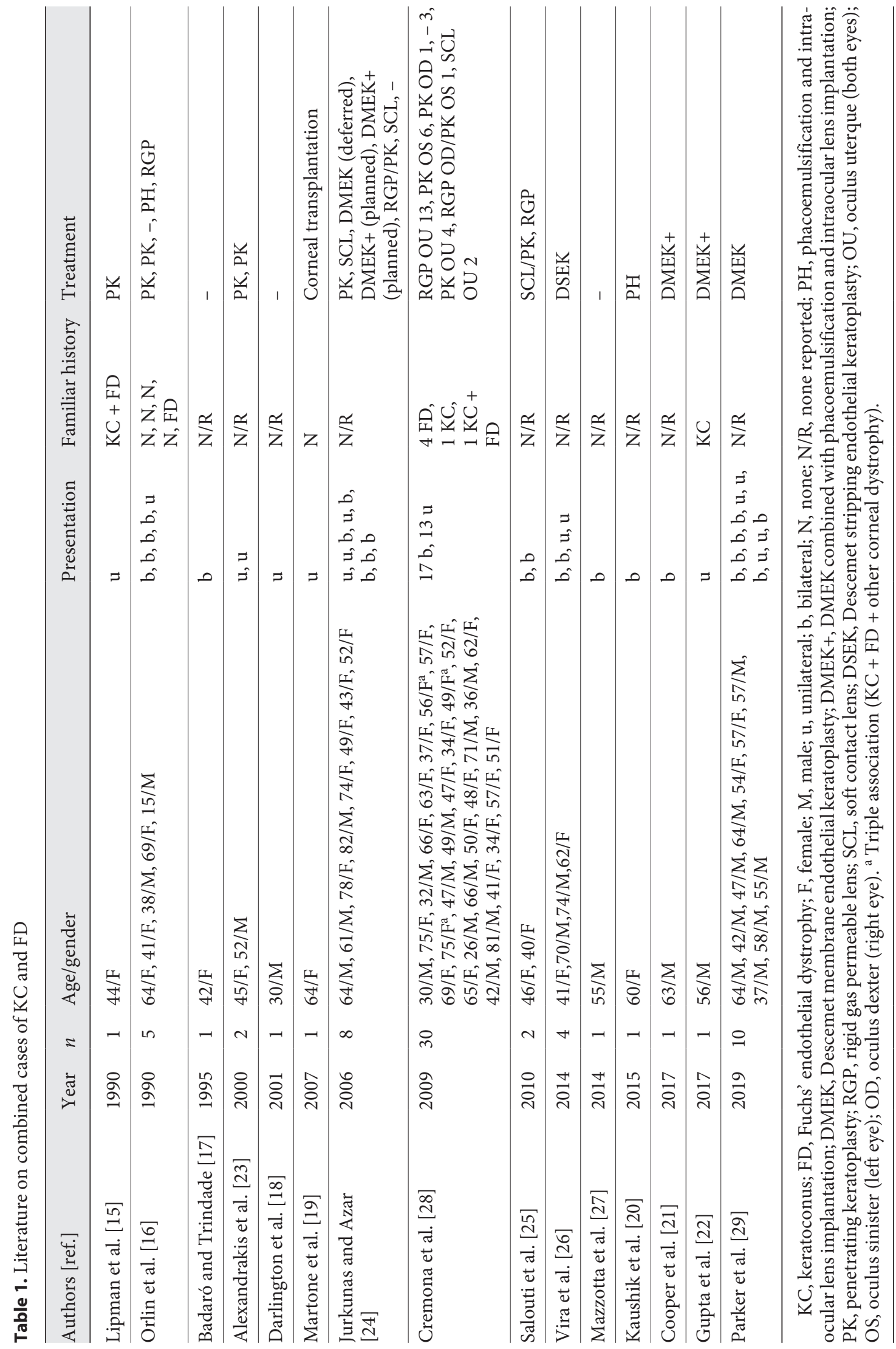


series [16, 20-27]. In the second category, there are 2 larger-scale studies, with Cremona et al. [28] expanding the scope to include all comorbid corneal dystrophies to KC found over a 10-year period in a specialized treatment center [28], while Parker et al. [29] expand the scope to address the mechanism for corneal hydrops in eyes with $\mathrm{KC}$, using a group of patients with $\mathrm{KC}$ and FD as 1 of the 2 research groups for their hypothesis.

\section{Discussion}

\section{Gender Predilection and Age Distribution}

The gender distribution is $39 / 69$ women, or $56.5 \%$, and $30 / 69$ men, or $43.5 \%$. In most cases $(41 / 69$, or $59.4 \%)$, there was bilateral involvement reported in the studies, either at the initial presentation or within a few years from it. This figure is likely to be higher, since initial unilateral presentations may have evolved to include the other eye a number of years after the respective study was published. The slightly higher percentage of cases among women may be attributable to the higher probability of females being diagnosed with FD, since $\mathrm{KC}$ does not have a clear gender predilection. The low total figure of patients does not permit for safe conclusions, the difference is not statistically significant, and examining the effect size shows that it is very small, $w=0.059$, indicating that there is very little, if any, clinical significance in the gender ratio. These calculations, however, are based on the small available sample and are subject to change.

Age range is very wide, from 15 to 82 years, but age is normally distributed, with a mean of 53.29 years and a median of 54 years (SD 14.23). Cases are either coming forward with a sight problem for the first time, in which case they are of younger age, or are discovered while being observed for a different eye condition, usually senile cataract or after receiving a graft. There was no statistically significant difference between the sexes for age. The wide age dispersion of cases is an interesting finding, since KC is typically diagnosed early in life. This either means that $\mathrm{KC}$ is initially expressed mildly or that the rarer cases of late $\mathrm{KC}$ development carry more risk for comorbid FD. Unfortunately, it is difficult to ascertain at which point in the patient's life these conditions developed, when the patient presents for examination.

\section{Incidence and Genetic Predisposition}

The absolute incidence of comorbid $\mathrm{KC}$ and FD based on the published studies cannot be directly assessed, since the reported cases spread over a very large period of time
(1990 until today), while individual case series include cases diagnosed over an extended time frame particular to each study. The most useful study in this respect is the retrospective study by Cremona et al. [28] in which the authors reported 27 cases of KC with FD and 3 cases of $\mathrm{KC}$ with FD combined with anterior membrane dystrophy within a 10-year time frame; the combined percentage among all cases of concomitant $\mathrm{KC}$ with any corneal dystrophy was $58.7 \%$ (30 out of 51 patients). Hence, we may conclude that FD is the most frequent comorbid corneal dystrophy diagnosed in KC patients; however, since the estimated prevalence for any of these conditions individually is low ( $\sim 1 \%$ for $\mathrm{KC}[2]$ and $\sim 0.1 \%$ for any dystrophy [30]), the combined incidence is expected to be approximately 1 per 100,000. The relative percentage of comorbid FD with $\mathrm{KC}$ among the instances of $\mathrm{KC}$ and comorbid dystrophies appears similar to the percentage of FD relative to other corneal dystrophies. It is thus reasonable to assume, until any experimental data or other epidemiological studies prove otherwise, that the combination of $\mathrm{KC}$ with FD is a randomly occurring event and the 2 conditions do not share common pathways in pathology. Unfortunately, the familial history is not recorded in most of the studies, and in those studies where it is recorded, there were 9 out of 38 cases with some relevant familial history; 5 cases with a history of FD, 2 cases with a history of KC, and 2 cases with a history of both conditions (in separate relatives).

\section{Comorbidity with Other Disorders}

Senile cataract is the most frequent comorbid condition, and it has in certain instances driven the patient to seek help, with KC and FD diagnosed subsequently [24]. There are also a few very rare instances of triple corneal dystrophy association - KC, FD, and epithelial basement membrane corneal dystrophy, 3 cases reported by Cremona et al. [28] and another case reported by Mazzotta et al. [27]; the latter paper mistakenly claims to have been the first such reference in the literature. Senile cataract does not share pathology with either KC or FD. Mazzotta et al. [27] reported the findings from a genetic study of the patient that revealed a mutation of the gene $Z E B 1$, a finding that is in accordance with a genetic study suggesting that the mutations are associated with $\mathrm{KC}$ and FD [31]. The rarity of this triple association, however, points to a random event.

\section{Potential Pathways of Disease}

There are 4 distinct possibilities mentioned in the literature for the cooccurrence of $\mathrm{KC}$ and FD. The first suggestion, by Darlington et al. [18], is the appearance of cor- 
neal guttata as a rare response to KC. This possibility has not been directly verified by any experimental studies so far, and there is no identified corneal disease encompassing both $\mathrm{KC}$ and corneal guttata.

A second possibility, put forward by Salouti et al. [25], is that of a single gene mutation, responsible for both conditions at the same time, possibly regulating the expression of other genes that have been associated with either one condition. This possibility has not been verified by any genetic studies, and one of the earliest related studies that reported an extensive familial history of related diseases has concluded that the most likely cause was the coinheritance of 2 distinct familial diseases rather than a single gene that regulates both [15]. A related third possibility raised by Salouti et al. [25] is that a disturbance in the terminal differentiation of the neural crest cells could be suspect, with both the corneal stroma and endothelium originating from the neural crest. This possibility has not been verified either.

Finally, the most likely possibility so far is chance, a possibility supported by the lower rate of incidence than one would expect should KC and FD be interlinked in some sort of causal manner, as discussed in the corresponding section above. Until genetic or histopathological studies conclusively prove otherwise, a chance cooccurrence of both diseases appears best supported by data. Naturally, we cannot exclude that $>1$ cause for the cooccurrence of $\mathrm{KC}$ and FD may exist, if another cause is aptly verified.

\section{Treatment}

Treatment varied depending on the initial presentation and the methods available at the time. The use of soft or rigid gas permeable lenses has resolved a small number of cases, while penetrating keratoplasty was employed in the early cases. Recent surgical developments include Descemet membrane endothelial keratoplasty and Descemet stripping automated endothelial keratoplasty, possibly combined with phacoemulsification and intraocular lens implantation if there is comorbid senile cataract.

\section{Clinical Importance}

The low incidence of KC with FD may at first glance lead the clinician to believe that the clinical significance would be low. However, Jurkunas and Azar [24] have warned against potential complications of cataract and refractive surgery in patients with $\mathrm{KC}$ and FD due to false normalization of the corneal pachymetry readings. Hence, clinicians should be wary of the possibility of cor- neal thinning caused by $\mathrm{KC}$ and concurrent increase in corneal thickness caused by FD. The authors suggested that routine use of preoperative topography and specular microscopy would minimize this risk. As mentioned, cataract was one of the most frequent comorbid conditions in senior patients with $\mathrm{KC}$ and $\mathrm{FD}$, and the rapid progression of cataract may mask any vision loss from $\mathrm{KC}$ and FD that would otherwise be diagnosed. However, the low probability of these disorders found in tandem reduces the overall risk.

\section{Conclusion}

This review of the rare instances of KC with FD demonstrated that the overall incidence of these disorders found in combination is very low and, in absence of other conclusively proven hypotheses, most likely attributable to chance. The treatment plan should be tailored to the needs of the individual patient, since the diagnosis can be made in any stage of life and could be part of a more complex presentation that includes another pathology of the cornea or senile cataract. The inclusion of preoperative topography and specular microscopy as routine in cataract preoperative assessment would protect against any potential complications in these patients.

\section{Statement of Ethics}

The authors have followed and support the Committee on Publication Ethics (COPE) guidelines on publishing ethics while writing this review article.

\section{Disclosure Statement}

The authors have no conflicts of interest to declare.

\section{Funding Sources}

No external funding was used in the study.

\section{Author Contributions}

I.M. performed the literature search and writing of the draft paper, I.T. contributed to study methodology and choice of included studies, while N.Z. and I.T. proofed the final manuscript. 


\section{References}

1 Galvis V, Sherwin T, Tello A, Merayo J, Barrera R, Acera A. Keratoconus: an inflammatory disorder? Eye (Lond). 2015 Jul;29(7): 843-59.

2 Gordon-Shaag A, Millodot M, Shneor E, Liu $Y$. The genetic and environmental factors for keratoconus. Biomed Res Int. 2015;2015: 795738.

3 Gordon-Shaag A, Millodot M, Essa M, Garth J, Ghara M, Shneor E. Is consanguinity a risk factor for keratoconus? Optom Vis Sci. 2013 May;90(5):448-54.

4 Tuft SJ, Hassan H, George S, Frazer DG, Willoughby CE, Liskova P. Keratoconus in 18 pairs of twins. Acta Ophthalmol. 2012 Sep; 90(6):e482-6.

5 Kok YO, Tan GF, Loon SC. Review: keratoconus in Asia. Cornea. 2012 May;31(5):581-93.

6 Fink BA, Wagner H, Steger-May K, Rosenstiel C, Roediger T, McMahon TT, et al. Differences in keratoconus as a function of gender. Am J Ophthalmol. 2005 Sep;140(3):45968.

7 Davidson AE, Hayes S, Hardcastle AJ, Tuft SJ. The pathogenesis of keratoconus. Eye (Lond). 2014 Feb;28(2):189-95.

8 Cristina Kenney M, Brown DJ. The cascade hypothesis of keratoconus. Cont Lens Anterior Eye. 2003 Sep;26(3):139-46.

9 Bawazeer AM, Hodge WG, Lorimer B. Atopy and keratoconus: a multivariate analysis. $\mathrm{Br} J$ Ophthalmol. 2000 Aug;84(8):834-6.

10 Li X, Rabinowitz YS, Rasheed K, Yang H. Longitudinal study of the normal eyes in unilateral keratoconus patients. Ophthalmology. 2004 Mar;111(3):440-6.

11 Han DC, Mehta JS, Por YM, Htoon HM, Tan DT. Comparison of outcomes of lamellar keratoplasty and penetrating keratoplasty in keratoconus. Am J Ophthalmol. 2009 Nov; 148(5):744-51.e1.

12 Klintworth GK. Corneal dystrophies. Orphanet J Rare Dis. 2009 Feb;4(1):7.
13 Vedana G, Villarreal G Jr, Jun AS. Fuchs endothelial corneal dystrophy: current perspectives. Clin Ophthalmol. 2016 Feb;10:321-30.

14 Wieben ED, Aleff RA, Tosakulwong N, Butz ML, Highsmith WE, Edwards AO, et al. A common trinucleotide repeat expansion within the transcription factor 4 (TCF4, E2-2) gene predicts Fuchs corneal dystrophy. PLoS One. 2012;7(11):e49083.

15 Lipman RM, Rubenstein JB, Torczynski E. Keratoconus and Fuchs' corneal endothelial dystrophy in a patient and her family. Arch Ophthalmol. 1990 Jul;108(7):993-4.

16 Orlin SE, Raber IM, Eagle RC Jr, Scheie HG. Keratoconus associated with corneal endothelial dystrophy. Cornea. 1990 Oct;9(4):299-304.

17 Badaró RM, Trindade FC. Ceratocone associado à distrofia endotelial de Fuchs: relato de um caso. Arq Bras Oftalmol. 1995;58:182-5.

18 Darlington JK, Mannis MJ, Segal WA. Anterior keratoconus associated with unilateral cornea guttata. Cornea. 2001 Nov;20(8):881-4.

19 Martone G, Tommasi C, Traversi C, Balestrazzi A, Berni E, Nuti E, et al. Unilateral corneal endothelial dystrophy and anterior keratoconus. Eur J Ophthalmol. 2007 MayJun;17(3):430-2.

20 Kaushik J, Jain AK, Jain VK, Chakma P. Phacoemulsification in a rare case of keratoconus with Fuch's endothelial corneal dystrophy. Int J Ophthalmol. 2015 Dec;8(6):1253-4.

21 Cooper E, Parker JS, Parker JS, Melles GR. Descemet membrane endothelial keratoplasty in an eye with Fuchs endothelial dystrophy and keratoconus. Ophthalmol Point Care. 2017;1:0000002.

22 Gupta R, Kinderyte R, Jacobs DS, Jurkunas UV. Elimination of anterior corneal steepening with Descemet membrane endothelial keratoplasty in a patient with Fuchs dystrophy and keratoconus: implications for IOL calculation. Cornea. 2017 Oct;36(10):1260-2.
23 Alexandrakis G, Filatov V, Adamis AP. Denovo development of corneal guttae and Fuchs' dystrophy in corneal grafts. CLAO J. 2000 Jan;26(1):44-6.

24 Jurkunas U, Azar DT. Potential complications of ocular surgery in patients with coexistent keratoconus and Fuchs' endothelial dystrophy. Ophthalmology. 2006 Dec; 113(12):2187-97.

25 Salouti R, Nowroozzadeh MH, Zamani M, Ghoreyshi M. Combined anterior keratoconus and Fuchs' endothelial dystrophy: a report of two cases. Clin Exp Optom. 2010 Jul; 93(4):268-70.

26 Vira S, Abugo U, Shih CY, Udell IJ, Sperling B, Hannush SB, et al. Descemet stripping endothelial keratoplasty for the treatment of combined fuchs corneal endothelial dystrophy and keratoconus. Cornea. 2014 Jan;33(1):1-5.

27 Mazzotta C, Traversi C, Raiskup F, Rizzo CL, Renieri A. First identification of a triple corneal dystrophy association: keratoconus, epithelial basement membrane corneal dystrophy and Fuchs' endothelial corneal dystrophy. Case Rep Ophthalmol. 2014 Sep;5(3):281-8.

28 Cremona FA, Ghosheh FR, Rapuano CJ, Eagle RC Jr, Hammersmith KM, Laibson PR, et al. Keratoconus associated with other corneal dystrophies. Cornea. 2009 Feb;28(2):127-35.

29 Parker JS, Birbal RS, van Dijk K, Oellerich S, Dapena I, Melles GR. Are Descemet membrane ruptures the root cause of corneal hydrops in keratoconic eyes? Am J Ophthalmol. 2019;205:204-5.

30 Musch DC, Niziol LM, Stein JD, Kamyar RM, Sugar A. Prevalence of corneal dystrophies in the United States: estimates from claims data. Invest Ophthalmol Vis Sci. 2011 Sep;52(9): 6959-63.

31 Lechner J, Dash DP, Muszynska D, Hosseini M, Segev F, George S, et al. Mutational spectrum of the ZEB1 gene in corneal dystrophies supports a genotype-phenotype correlation. Invest Ophthalmol Vis Sci. 2013 May;54(5): 3215-23. 